Psychotherapeut 2022 67:116-121

https://doi.org/10.1007/s00278-022-00573-2

Angenommen: 10. Januar 2022

Online publiziert: 16 . Februar 2022

(c) Der/die Autor(en) 2022

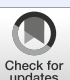

Check for
updates

\section{In diesem Beitrag}

- Merkmale pathologischer Narrative Kohärenz • Agency • Perspektiven

- Resümee

\title{
Wie Erzählungen Hinweise auf psychische Störungen geben können
}

\author{
Lisa Pfeifer \\ Arbeitsbereich Psychoanalyse, Institut f. Psychologie, Goethe-Universität Frankfurt, Frankfurt am Main, \\ Deutschland
}

\section{Zusammenfassung}

Als Ausgangspunkt dieser Arbeit dienen Ansätze, die eine narrative Perspektive für das Verständnis von Psychopathologie und die psychotherapeutische Praxis vorschlagen. Im Hinblick auf die Fragen, welche Vorteile die Analyse von Patient*innenerzählungen bieten kann, und durch welche Merkmale psychopathologische Narrative sich auszeichnen, wird ein Überblick über ausgewählte Fallberichte, empirische Untersuchungen und theoretische Überlegungen gegeben. Diese werden unter den drei Kategorien Kohärenz, „Agency“ und Perspektiven beschrieben. Die Arbeit mag einen Impuls geben, ein tieferes Verständnis für narrative Dysfunktionen zu entwickeln und ihre Ursprünge sowie ihre Bedeutung für psychische Störungen und deren Behandlung vermitteln.

Schlüsselwörter

Narrative Analyse · Erinnerung · Emotionen · Selbstkonzept · Psychopathologie

Es ist anzunehmen, dass Psychotherapeut*innen intuitive Einschätzungen über die Sprache und Erzählweise ihrer Patient*innen vornehmen. Eine qualitative Untersuchung von ausgewählten Erzählungen kann Aufschluss über die Verarbeitung emotionaler Erlebnisse und die Denkorganisation der Patient*innen geben. Weiterhin eröffnen sich so $\mathrm{Zu}$ sammenhänge zwischen der Form autobiografischer Erzählungen und dem Krankheitserleben.

\section{Einleitung}

Der Mensch ist prädisponiert, Erfahrungen in einer narrativen Form mit Absichten und Handlungsplot zu organisieren. In einem narrativen Denkmodus versucht er, sich den Rätseln menschlichen Verhaltens anzunähern. Nicht die "Wahrheit", sondern eine realistische Repräsentation des Lebens ist das Ziel:

Es gibt, psychologisch gesehen, kein „Leben an sich". Geringstenfalls ist es ein selektives Aufrufen von Erinnerungen, darüber hinaus ist das
Erzählen des eigenen Lebens eine interpretative Leistung. (Bruner 1987, S. 13; Übersetz. L.P.)

Autobiografische Erzählungen sind bedeutsam für die Entwicklung der eigenen Identität (Habermas und Kemper 2021; Linde 1993). Dabei wird eine Wechselwirkung zwischen Selbstkonzept, der dazugehörigen aktuellen Lebenserzählung und der Art und Weise, wie Ereignisse erlebt und anschließend in der Lebenserzählung verarbeitet und interpretiert werden, angenommen (McLean et al. 2007). Diesem Ansatz folgend kann Psychopathologie auch als Störung des linguistisch und narrativ organisierten Bedeutungssystems verstanden werden (Gonçalves et al. 2002). Die Analyse von Erzählungen kann zu einem tieferen Verständnis davon führen, wie Psychopathologie in der Sprache ausgedrückt wird. Auch das Selbsterleben von Patient*innen erfährt man am zugänglichsten über ihre Narrative (Soroko 2013). Es wurde lange übersehen, dass Sprache und Erzählungen selbst Ausdruck von Psychopathologie sein können (Capps und Ochs 1995). Nicht nur der 
Inhalt von Erzählungen gibt Aufschluss über Symptome und das (Nicht-)Zutreffen von ICD-Kriterien: Narrative sind auch in ihrer Form, z. B. in dem, wie sie und was sie nicht erzählen, unmittelbar mit dem Berichteten vereint und von ähnlicher Bedeutung für die Psychotherapie (Schafer 1995). Der Fokus der Psychologie auf nomothetische Ansätze übersieht daher allzu oft die verflochtene Problematik von Sprache, Bedeutung und Kontext (Mishler 1986). Narrative sollten in der klinischen Praxis also nicht einfach als Mittel benutzt werden, um an einen zugrunde liegenden chronologischen Bericht der Geschehnisse zu gelangen, sondern selbst als psychologisches Phänomen betrachtet werden (Soroko 2013).

Es erscheint schlüssig, dass die Fähigkeit, Ereignisse zu erzählen, eng mit der Verfügbarkeit eines psychischen Raums verknüpft ist, in dem die Realität symbolisiert werden kann, Gedanken und Gefühle als innere Phänomene verstanden werden und gleichzeitig anerkannt wird, dass diese mit äußeren Ereignissen in Beziehung stehen (Fonagy und Target 2001). Werden Gedanken und Gefühle aber als unmittelbar auf die Realität einwirkend wahrgenommen, erschrecken sie ihre*n Urheber*in und können in ihrer Bedeutung überbewertet werden. Eine solche Entwicklung erschwert es, die eigenen Regungen mit der äußeren Realität in Einklang zu bringen, sie angemessen zu symbolisieren (Fonagy und Target 2001) und in Form einer Geschichte erfahren und interpretieren zu können (Habermas 2019).

Eine narrative Perspektive gibt es spätestens seit den 1980er-Jahren in der psychotherapeutischen und v.a. psychodynamisch-orientierten Praxis. So kann es eine Aufgabe in der Therapie sein, eine Lebenserzählung zu entwickeln, die es den Patient*innen erlaubt, die Ursprünge, Bedeutung und Relevanz ihrer aktuellen Probleme so zu verstehen, dass sie sie verändern können (Schafer 1983). Ein Repräsentant ist Ferro (2005), der die Psychoanalyse als Therapie durch Geschichtenerzählen beschreibt und sich theoretisch auf die Überlegungen zur $a$-Funktion (Bion 1962) bezieht. Er postuliert, dass die meisten Psychopathologien auf eine Störung der a-Funktion, also letztendlich die Störung, Eindrücke zu erzählbaren Ge- danken umzuwandeln, zurückgeführt werden können. Gemäß Ferro übertragen Patient*in und Therapeut*in unartikulierte Emotionen und Bedürfnisse auf das bipersonelle Feld. Hierbei treten sie in Dialog und schaffen gemeinsam Bedeutung aus den im Feld befindlichen Elementen. Auch gibt es konstruktivistische Therapieansätze (u.a. White und Epston 1990). Hierbei werden problematische Elemente der Lebenserzählung identifiziert, um anschließend eine alternative, besser zu der eigenen Identität passende Erzählung zu rekonstruieren.

Auf Grundlage dieser theoretischen Ausführungen über die narrative Beschaffenheit des menschlichen Denkens wird im Folgenden argumentiert, dass Psychopathologie zumindest in Teilen als Störung des narrativen Organisationssystems verstanden werden kann. Wenn nun über eine narrative Analyse nachvollzogen werden kann, wie Menschen ihre Erzählungen organisieren, lässt sich daraus schließen, wie sie das Erlebte interpretiert und verarbeitet haben (Bruner 1987). Das hilft zum einen, die Psyche, und zum anderen, das tatsächlich Erlebte besser zu verstehen. Ziel der Untersuchung ist die Identifizierung formaler Kriterien autobiografischer Erzählungen, die auf eine Psychopathologie hinweisen können.

\section{Merkmale pathologischer Narrative}

Der Unglückliche ist nun derjenige, der sein Ideal, seinen Lebensinhalt, die Fülle seines Bewußtseins, sein eigentliches Wesen irgendwie außer sich hat. Der Unglückliche ist immer sich abwesend, nie sich selbst gegenwärtig. (Kierkegaard 1988, S. 259)

Grundlage des Beitrags sind theoretische Überlegungen zu funktionalen und nichtfunktionalen Narrativen. Diese basieren auf ausgewählten Fallbeispielen, die sich den Erzählweisen der Patient*innen widmen, sowie ausgewählten empirischen Studien, die Erzählungen in klinischen Stichproben untersuchen.

Die Auswahl der drei Kategorien Kohärenz, „Agency“ und Perspektiven beruht auf der klinischen Relevanz dieser Charakteristiken in den autobiografischen Erzählungen von Patient*innen. Sie erfüllen spezifische Funktionen in Erzählungen und können Hinweise auf die Psychopathologie geben.

Kohärenz beschreibt verschiedene Dimensionen, in denen die Teile einer Erzählung in einen Zusammenhang gesetzt werden können und somit eine sinnvolle und nachvollziehbare Geschichte ergeben. Die klinische Bedeutung einer kohärenten autobiografischen Erzählung zeigt sich u.a. im Adult-Attachment-Interview. Dort wird sie als Hinweis auf die innere Repräsentation von Bindungserfahrungen erfasst (George et al. 1996). Darüber hinaus ist die Diskontinuität von Erzählungen mit Identitätsverwirrung (Erikson 1974) und Borderline-Persönlichkeitsstörungen assoziiert (Kernberg 2018).

Mit "Agency" ist das Ausmaß gemeint, in dem ein Individuum erlebt, Einfluss auf sich und seine Umwelt ausüben zu können. Das Erleben des Selbst als von äußeren Einflüssen geleitet, lässt sich in Lebenserzählungen ohne einflussreiche*n Protagonist*in erkennen und steht beispielsweise mit dem depressiven Attributionsstil oder Neurosen (Schafer 1983) im Zusammenhang.

Perspektiven in Erzählungen beinhalten sowohl die Beschreibung von Emotionen als auch thematische Vielfalt und die Fähigkeit, einzelne Ereignisse aus verschiedenen Blickwinkeln betrachten zu können. Eine Einschränkung von Perspektiven gibt Hinweis auf die Abwehr von Gefühlen und Einsichten (Habermas 2019) und ist u.a. mit Alexithymie (Dimaggio 2011) assoziiert.

\section{Kohärenz}

Die Fähigkeit, etwas narrativ zu strukturieren, entwickelt sich aus dem Bedürfnis, Ambiguität zu reduzieren und Kohärenz zu schaffen (Mancuso 1986). Der Mechanismus, chaotische Einflüsse von innen und außen in ein kohärentes Narrativ einzuordnen, stellt die Grundlage des menschlichen Bedeutungsschaffen dar und ist essenziell für das psychische Überleben (Gonçalves et al. 2002). So legen Studien einen positiven Zusammenhang von narrativer Kohärenz und psychischem Wohlbefinden nah: Bei Jugendlichen, die eine Wendepunkterzählung aufschrieben und Fragebogen zu psychischem 
Wohlbefinden und psychopathologischen Symptomen ausfüllten, war kausale Kohärenz sowohl aktuell als auch im Abstand von einem Jahr mit höherem Wohlbefinden assoziiert (Mitchell et al. 2020; Adler et al. 2016).

Der Fall der 18-jährigen, an Hysterie leidenden Patientin Dora von Freud (1993) gibt einen ersten Hinweis auf den Zusammenhang von inkohärenten Erzählungen und psychischem Leiden. Die Erzählungen über ihr Leben und ihre Krankheit waren oftmals orientierungslos; vieles blieb verborgen und unklar. Aus ihrer Geschichte lässt sich schlussfolgern, dass das menschliche Leben im Idealfall als verbundene und kohärente Erzählung begriffen werden kann, in der verschiedene Episoden in einen motivationalen Zusammenhang eingebettet sind. Eine psychische Störung kann demzufolge zumindest in Teilen als das Leiden an einer inkohärenten Lebensgeschichte verstanden werden, die das Selbst nicht adäquat erfasst (Marcus 1981). Denn, wenn das Selbst narrativ begriffen wird, entsteht es auf der Grundlage von Ereignissen und Erlebnissen, die bedeutungsvoll in die Lebensgeschichte integriert werden und somit das Selbstkonzept prägen (Habermas und Bluck 2000). Diese Wechselwirkung ermöglicht, mit Erlebnissen umzugehen, die das Selbstverständnis herausfordern und sich an neue Umstände anzupassen. In einer Studie, die den Zusammenhang von autobiografischen Erzählungen über persönlich bedeutsame Ereignisse und psychischem Wohlbefinden sowie Ausführungen zur eigenen Identitätsentwicklung zum Inhalt hat, zeigte sich, dass die Fähigkeit, die eigene Identitätsentwicklung kohärent erzählen zu können, mit einem Gefühl von Bestimmung und Sinnhaftigkeit assoziiert ist (Waters und Fivush 2015).

Eine kohärente Lebenserzählung erfüllt also die individuelle Funktion, das eigene Selbstbild zu etablieren. Aber sie ermöglicht auch den sozialen Austausch, weil die eigenen Erfahrungen anderen verständlich kommuniziert werden können (Dimaggio und Semerari 2001). Salvatore et al. (2004) entwerfen ein Modell, in dem sie strukturelle Schwächen von Erzählungen auf einer protonarrativen Ebene verorten. Auf dieser Stufe werden mentale Bilder mit Emotionen verknüpft, um später in eine bedeutsame Erzählung integriert zu werden. Bei schweren Persönlichkeitsstörungen ist zu beobachten, dass die Verknüpfung von Erlebnissen und Affekten dysfunktional ist: Starke Emotionen evozieren unkontrolliert Erinnerungen, die nicht sinnvoll miteinander in Beziehung gesetzt werden können. Die entstehenden ungeordneten Narrative machen es den Zuhörenden häufig unmöglich zu dechiffrieren, was die Erzählenden aussagen möchten.

Neben der beschriebenen kommunikativen Funktion ermöglichen Erzählungen einen Blick auf das eigene Leben als temporalen Verlauf von Vergangenheit, Gegenwart und Zukunft. So betrachtet, hat auch das Entwickeln von Hoffnung Kohärenz zur Voraussetzung: Sie ermöglicht die Vorstellung einer Zukunft, die dem fortlaufenden Leben Sinn verleiht. Der Zusammenhang von Hoffnungslosigkeit und Psychopathologie lässt sich in den Narrativen von Patient*innen mit Schizophrenie beobachten (Keen 1986): Sie erlauben kein Neues, da sie bereits formuliert sind und alles Erlebte mit schon vorhandenen Begriffen beschreiben. Keen (1986) bezeichnet diese Art der Erzählung als kataklysmisch: Es kann keine Zukunft gedacht werden, in der das Gute das Böse besiegt und das Selbst geheilt wird. Diese temporale Komponente erinnert an Narrativität an sich, also dass die Geschichte zeitlich und inhaltlich fortschreitet, statt sich in Wiederholungen und Gedankenschleifen zu verlieren. Auch depressive Störungen hängen hiermit zusammen, denn sie zeichnen sich u. a. durch eine Beeinträchtigung des subjektiven Zeitgefühls aus: Die Untersuchung einer klinischen Stichprobe zeigte, dass sich depressive Personen in ihren Lebenserzählungen mehr auf die Vergangenheit beziehen und häufiger von einer linearen Erzählform abweichen (Habermas et al. 2008). Charakteristisch ist die Wahrnehmung, dass die Zeit stillstehen würde, die Vergangenheit auch schon schlecht gewesen sei und sich daran auch in Zukunft nichts ändern wird. Das wird in depressiven Narrativen reflektiert, sie sind ruminativ und weniger linear als die von nichtdepressiven Menschen (Habermas et al. 2008).

Es lässt sich festhalten, dass Kohärenz dem Selbst Flexibilität verleiht, weil sie einschneidende Ereignisse in die Lebens- erzählung integriert und Aussicht auf eine Zukunft ermöglicht. Jedoch können Erzählungen auch kohärent strukturiert und nachvollziehbar erzählt, aber trotzdem pathologisch sein (Salvatore et al. 2004). Solche maladaptiven Formen von Kohärenz werden weiter unten aufgegriffen.

\section{Agency}

Im Kleinkindalter machen die Menschen die Erfahrung, dass die Außenwelt und ihre Mitmenschen durch das eigene Handeln beeinflusst werden können. Es entwickelt sich die Gewissheit, selbst Urheber seiner Gedanken und Taten zu sein. Die Wahrnehmung der eigenen Handlungsfähigkeit ist grundlegend für ein gesundes Vertrauen in sich selbst (Stern 1977). Ein Mangel an „Agency" kann dagegen zum Verlust des Identitätsgefühls führen, womöglich entsteht sogar der Eindruck, von fremden Kräften gelenkt zu werden (Dimaggio 2011).

Fehlende "Agency" in Narrativen kann durch bestimmte semantische Formen identifiziert werden. Schafer (1983) beobachtete, dass neurotische Patient*innen häufig die eigene Involvierung abstreiten (,Meine Erwartung an den heutigen Tag hat mich nicht schlafen lassen“). Diese Sprache der Leugnung vergleicht er mit der konkretistischen Gefühlswelt eines kindlichen, körperzentrierten Selbst und beschreibt sie als Abwehrmechanismus: Indem Patient*innen ihre Handlungen nicht auf sich selbst beziehen, können sie verstörende Affekte und Motivationen abwehren. Als Gegensatz entwirft Schafer die Sprache der Handlung, die das Selbst als Verursacher darstellt. Seines Erachtens bedeutet Fortschritt in der Therapie, dass der oder die Patient*in sich nach und nach als verantwortlich für die eigenen Handlungen begreift und die Autor*innenschaft der eigenen Lebensgeschichte übernimmt.

Patient*innen mit unterschiedlichen Persönlichkeitsstörungen berichten zwar von Entscheidungen und Handlungen, begreifen diese aber nicht als selbstinitiiert. Das resultierende Gefühl des Ausgeliefertseins ist entscheidend für ihren Leidensdruck (Dimaggio 2011). Auch der für Depressionen typische Attributionsstil verweist auf eine maladaptive Wahrnehmung der eigenen „Agency“. So schreiben 
an Depressionen Erkrankte negative Ereignisse häufig ihren eigenen Handlungen zu, während sie Positives als zufällig attribuieren (Habermas 2015). In einer umfangreichen Analyse der Geschichten einer unter Agoraphobie leidenden Patientin beschreiben Capps und Ochs (1995) eine Erzählerin, die sich selbst als hilflose Protagonistin konstruiert. Die Patientin schafft ein Narrativ, das von Gefahr und Kontrollverlust gekennzeichnet ist. Sie beschreibt sich selbst konsequent in anderen Rollen als die der Handelnden. Dabei benutzt sie Modalverben, die ihre Handlungen als notwendig und nicht als freiwillig darstellen. Indem sie ihr Scheitern in den Vordergrund rückt, betont sie ihre Verletzlichkeit und spricht sich die Fähigkeit $a b$, etwas bewältigen und kontrollieren zu können.

Zusammenfassend scheint die Anerkennung der eigenen „Agency“ grundlegend dafür zu sein, sich als einflussreiche ${ }^{*} n$ Protagonist*in der eigenen Lebenserzählung wahrzunehmen. Daraus lassen sich wichtige therapeutische Implikationen ableiten. Schließlich setzt die Motivation, etwas verändern zu wollen, die Annahme voraus, dass man sein Leben positiv und negativ beeinflussen kann.

\section{Perspektiven}

Die Entwicklung der Reflexionsfähigkeit, also sich beobachten, reflektieren und korrigieren zu können, ist eng damit verbunden, über sich selbst zu erzählen. Dafür muss das eigene Leben aus verschiedenen Perspektiven betrachtet werden. Dieser Prozess ist grundlegend für ein funktionales Selbst (Linde 1993).

Eine mangelhafte Reflexionsfähigkeit führt zu einer Einschränkung von Perspektiven in Erzählungen. Der Verzicht auf Perspektiven kann aber auch dazu dienen, bedrohliche Emotionen, Regungen und Erinnerungen abzuwehren. So können Konflikte und Ambivalenz vermieden werden (Habermas 2019). Sowohl das Auslassen von Emotionen und verschiedenen Blickwinkeln als auch thematische Zuspitzungen können Merkmale eines pathologischen Narrativs sein.

Neben Narrativen mit strukturellen Defiziten weisen „verarmte Narrative“ auf Persönlichkeitsstörungen hin (Dimaggio
2011). Diese Beobachtung beruht auf der Arbeit mit Patient*innen, die sich schwertun, autobiografische Details zu erzählen. Stattdessen treffen sie abstrakte theoretische Aussagen, um Probleme zu kommunizieren. Verarmten Narrativen fehlt es an Details, bildlicher Qualität und der Beschreibung von Emotionen. In ihrem „referential process model" benennt Bucci (2011) kognitive Prozesse, durch die sich der emotionale Zugang zu Erlebtem verändert. Die hier beschriebene narrative Entwicklung vollzieht sich von einer verarmten Erzählung, in der schmerzhafte Affekte auf körperlicher und motorischer Ebene ausgedrückt werden, hin zur Identifikation von Emotionen und der Fähigkeit, diese zu symbolisieren. Sie wird durch die containende Beziehung von Therapeut*in und Patient*in angeregt.

Bei der dissoziativen Identitätsstörung sind narrative Perspektiven von besonderer Bedeutung. Hierbei wechseln $\mathrm{Pa}$ tient*innen zwischen verschiedenen Identitäten, die jeweils eigene Wahrnehmungen, Emotionen und Erinnerungen haben. Der Krankheit liegt aus psychodynamischer Perspektive eine Spaltung zugrunde, die frühe traumatische Ereignisse abwehren soll. Beran und Unoka (2005) beschreiben beispielhaft den Psychotherapieverlauf einer Patientin, deren Selbstnarrativ in mehrere Identitäten fragmentiert ist. Dem Therapeuten ist es im Verlauf der Therapie gelungen, die narrative Perspektive der Patientin zu verändern, indem er die voneinander isolierten Erzählungen miteinander verbunden hat. Hierfür hat er etwa die Rolle einer ihrer Identitäten im Dialog übernommen und die verschiedenen Sichtweisen der Patientin miteinander in Bezug gesetzt. So konnte die „Hauptidentität“ der Patientin auf zuvor unzugängliche Erinnerungen zugreifen und ein vollständigeres Narrativ entwickeln. Auch gelang es der Patientin, die narrative Perspektive des Therapeuten zu übernehmen und so ein neues, verbundenes Selbst durch die dialogische Interaktion zu konstruieren.

Als bestimmte Form eingeschränkter Perspektiven kann auch „Hyperkohärenz" (Gonçalves et al. 2002) auf psychische Störungen hinweisen. Patient*innen mit Schizophrenie können häufig nur sehr begrenzt Erzählungen generieren, um Neues zu beschreiben. Da alles an bereits formu- lierte Erzählungen angepasst wird, ist die Verarbeitung neuer Erfahrungen eingeschränkt (Keen 1986). Daraus resultiert eine fatalistische Weltanschauung (Gonçalves et al. 2002). Hierunter fallen außerdem sog. kontaminierte Narrative, in denen alle Ereignisse, ob glücklich oder traurig, im Laufe der Erzählung zu einem negativen Ende finden. Die negative Verarbeitung emotionaler Erlebnisse steht mit ruminativem Denken und Selbstvorwürfen in Verbindung und findet sich häufig in den Erzählungen depressiver Patient*innen wieder (Cox und McAdams 2014). Auch die von Capps und Ochs (1995) dargestellten Erzählungen einer Patientin, die unter Agoraphobie leidet, erfüllen die thematische Kohärenz in einem Ausmaß, das die Sicht auf die Ereignisse einschränkt und Neubewertungen unmöglich macht. Ihr subjektives Zeitgefühl orientiert sich an einem prägenden Panikereignis. Anstatt ihre Kompetenzen anzuerkennen, beschreibt sie ihr damals souveränes Verhalten als präagoraphobisch und bringt frühere Unsicherheiten vorausahnend mit ihrer jetzigen Angststörung in Verbindung. So erscheint diese als vorherbestimmt und unausweichlich.

Die obigen Ausführungen verdeutlichen die Aktualität von Freuds Konzept des Durcharbeitens. Eine möglichst detaillierte, perspektivenreiche Erinnerung, die symbolisiert und in Worte gefasst wird, ermöglicht das Bewusstwerden und Verstehen von Verdrängtem, das dann reflektiert und in die Erzählung integriert werden kann (Habermas 2015).

\section{Resümee}

In dieser Arbeit wurde davon ausgegangen, dass Psychopathologie mit der Störung narrativer Fähigkeiten in Beziehung steht und die Analyse von Erzählungen dem tieferen Krankheitsverständnis dienen kann. Es hat sich gezeigt, dass eine mangelhafte Erzählfähigkeit auf unterschiedliche Weise in den autobiografischen Narrativen von Patient*innen zum Ausdruck kommt. Noch scheint die Forschung nicht so weit zu sein, um eine detaillierte Anleitung zur narrativen Diagnostik zu entwerfen. Ansätze lassen sich jedoch u. a. bei Soroko (2013) oder Salvatore et al. (2004) erkennen. Weiterführend lassen sich beispielsweise Veränderungen 
im Laufe einer Therapie hin zu einer kohärenten, agentischen und integrierenden Erzählung prüfen, um eine diagnostische Aussage zu treffen. In dieser Arbeit wurde sich auf die Struktur und Erzählweise in autobiografischen Narrativen konzentriert. Weitere interessante Kriterien, wie inhaltliche Auswertungen, die Beziehung von Erzählungen zu Symptomen und die lebensgeschichtliche Entwicklung von Störungsbild und Narrativ sollten ebenfalls beachtet werden, um ein umfassendes Bild der Zusammenhänge zwischen Narration und psychischen Störungen zu erhalten.

\section{Fazit für die Praxis}

- Erzählungen dienen nicht allein der Kommunikation von Symptomen; sie selbst sollten als psychologisches Phänomen betrachtet werden. Diese Arbeit bietet einen Ansatz dafür, welche Merkmale von Patient*innennarrativen genauer analysiert werden können, um ein tieferes Krankheitsverständnis zu erlangen.

- Die Analyse von autobiografischen Narrativen ist insofern diagnostisch wertvoll, als sie Aufschluss über das Selbsterleben und die Konstruktion der Erfahrungswelt der Patient*innen gibt. Weiterhin lassen sich der Psychopathologie zugrunde liegende Abwehrmechanismen ableiten.

- Die gemeinsame Konstruktion eines funktionalen Narrativs von Patient*in und Therapeut*in kann als Teil einer erfolgreichen Psychotherapie gelten.

\section{Korrespondenzadresse}

\section{Lisa Pfeifer}

Arbeitsbereich Psychoanalyse, Institut $f$. Psychologie, Goethe-Universität Frankfurt Theodor-W.-Adorno-Platz 6, 60629 Frankfurt am Main, Deutschland s8990418@stud.uni-frankfurt.de

Funding. Open Access funding enabled and organized by Projekt DEAL.

\section{Einhaltung ethischer Richtlinien}

Interessenkonflikt. L. Pfeifer gibt an, dass kein Interessenkonflikt besteht.

Für diesen Beitrag wurden von der Autorin keine Studien an Menschen oder Tieren durchgeführt. Für die aufgeführten Studien gelten die jeweils dort angegebenen ethischen Richtlinien.

Open Access. Dieser Artikel wird unter der Creative Commons Namensnennung 4.0 International Lizenz

\section{How narratives can give hints towards psychological disorders}

Attempts to propose a narrative perspective for the understanding of psychopathology and psychotherapeutic practice serve as the starting point of this paper. With respect to the questions, namely which are the benefits provided by the analysis of patients' narratives and which are the features that are characteristic for psychopathological narratives, an overview of selected case studies, empirical investigations and theoretical considerations is given. The findings can be summarized under the three terms of coherence, agency and perspectives. This paper is expected to provide an impulse to convey a deeper understanding of narrative dysfunctions, their origins and their importance for psychological disorders and their treatment.

\section{Keywords}

Narrative analysis · Memory · Emotions · Self concept · Psychopathology veröffentlicht, welche die Nutzung, Vervielfältigung, Bearbeitung, Verbreitung und Wiedergabe in jeglichem Medium und Format erlaubt, sofern Sie den/die ursprünglichen Autor(en) und die Quelle ordnungsgemäß nennen, einen Link zur Creative Commons Lizenz beifügen und angeben, ob Änderungen vorgenommen wurden.

Die in diesem Artikel enthaltenen Bilder und sonstiges Drittmaterial unterliegen ebenfalls der genannten Creative Commons Lizenz, sofern sich aus der Abbildungslegende nichts anderes ergibt. Sofern das betreffende Material nicht unter der genannten Creative Commons Lizenz steht und die betreffende Handlung nicht nach gesetzlichen Vorschriften erlaubt ist, ist für die oben aufgeführten Weiterverwendungen des $\mathrm{Ma}$ terials die Einwilligung des jeweiligen Rechteinhabers einzuholen.

Weitere Details zur Lizenz entnehmen Sie bitte der Lizenzinformation auf http://creativecommons.org/ licenses/by/4.0/deed.de.

\section{Literatur}

Adler JM, Lodi-Smith J, Philippe FL, Houle I (2016) The incremental validity narrative identity in predicting well-being: a review of the field and recommendations for the future. Pers Soc Psychol Rev 20(2):142-175

Beran E, Unoka Z (2005) Construction of self-narrative in a psychotherapeutic setting. In: QuasthoffUM, Becker T (Hrsg) Narrative interaction. Benjamins, Amsterdam, S151-167

Bion W (1962) A theory of thinking. Int J Psychoanal 43:306-310

Bruner J (1987) Life as narrative. Soc Res 51(1):11-32

Bucci W (2011) The role of subjectivity and intersubjectivity in the reconstruction of dissociated schemas; converging perspectives from psychoanalysis, cognitive science and affective neuroscience. Psychoanal Psychol 28(2):247-266

Capps L, Ochs E (1995) Constructing panic: the discourse of agoraphobia. Harvard University Press, Cambridge

Cox K, McAdams DP (2014) Meaning making during high and low point of life story episodes predicts emotion regulation two years later: how the past informs the future. J Res Pers 50:66-70

Dimaggio G (2011) Impoverished self-narrative and impaired self-reflection as targets for the psychotherapy of personality disorders. JContemp Psychother 41(3):165-174

Dimaggio G, Semerari A (2001) Psychopathological narrative forms. JConstr Psychol 14:1-23

Erikson EH (1974) Jugend und Krise: Die Psychodynamikim sozialen Wandel. Klett, Stuttgart

Ferro A (2005) Psychoanalysis as therapy and storytelling. Routledge, London, New York

Fonagy P, Target M (2001) Mit der Realität spielen. Zur Doppelgesichtigkeit psychischer Realität von Borderline-Patienten. Psyche 55(9-10):961-995

Freud S (1993) Bruchstück einer Hysterie-Analyse. Fischer, Frankfurt

George C, Kaplan N, Main M (1996) Adult Attachment Interview; unveröffentlichtes Manuskript. Department of Psychology, University of California, Berkeley

Gonçalves OF, Machado PPP, Korman Y, Angus L (2002) Assesing psychopathology: a narrative approach. In:BeutlerLE, MalikML (Hrsg) Rethinking the DSM: a psychological perspective. American Psychological Association, Washington, S149-176

Habermas T (2015) A model of psychopathological distortions of autobiographical memory narratives: an emotion narrative view. In: Watson LA, Bernsten D (Hrsg) Clinical perspectives on autobiographical memory. Cambridge University Press, Cambridge, S267-290

Habermas T (2019) Emotion and narrative: perspectives in autobiographical storytelling. Cambridge University Press, Cambridge

Habermas T, Bluck S (2000) Getting a life. The emergence of the life story in adolescence. Psychol Bull 126(5):748-769

Habermas T, Kemper N (2021) Psychoanalytic perspectives on identity: from ego to life narrative. In: Bamberg M, Demuth C, Watzlawik M (Hrsg) The Cambridge handbook of identity. Cambridge University Press, Cambridge, S 193-214

Habermas T, Ott L-M, Schubert M, Schneider B, Pate A (2008) Stuck in the past: negative bias, explanatory style, temporal order, and evaluative perspectives in life narratives of clinically depressed individuals. Depress Anxiety 25(11):121-132

Keen E (1986) Paranoia and cataclysmic narratives. In: Sarbin TR (Hrsg) Narrative psychology: the storied nature of human conduct. Praeger, New York, S174-192

Kernberg OF (2018) Treatment of severe personality disorders. APA, Washington 
Kierkegaard S (1988) Entweder - Oder. Teil 1. DTV, München (FauteckH, Übersetz.)

Linde C (1993) Life stories: the creation of coherence. Oxford University Press, New York

Mancuso JC (1986) The acquisition and use of narrative grammar structure. In: Sarbin TR (Hrsg) Narrative psychology: the storied nature of human conduct. Praeger, New York, S 91-110

Marcus S (1981) Freud and Dora: story, history, case history. In: Meisel P (Hrsg) Freud: a collection of critical essays. Prentice Hall, New Jersey, S183-210

McLean KC, Pasupathi M, Pals JL (2007) Selves creating stories creating selves: a process model of self-development. Pers Soc Psychol Rev 11(3):262-278

Mishler EG (1986) The analysis of interview-narratives. In: Sarbin TR (Hrsg) Narrative psychology: the storied nature of human conduct. Praeger, New York, S233-255

Mitchell C, Reese E, Salmon K, Jose P (2020) Narrative coherence, psychopathology, and wellbeing: congruent longitudinal findings in a midadolescent sample. J Adolesc 79:16-25

Salvatore G, Dimaggio G, Semerari A (2004) A model of narrative development: Implications for understanding psychopathology and guiding therapy. Psychol Psychother Theory Res Pract 77(2):231-254

Schafer R (1983) The analytic attitude. Basic Books, New York

Schafer R (1995) Erzähltes Leben: Narration und Dialog in der Psychoanalyse. Pfeiffer, München

Soroko E (2013) Self-narrative analysis methods in clinical diagnosis: the example of paranoid personality disorder. Ann Psychol XVI(1):37-62

Stern DN (1977) The first relationship. Open Books, London

Waters TEA, Fivush R (2015) Relations between narrative coherence, identity, and psychological well-being in emerging adulthood. J Pers 83(4):441-451

White M, Epston D (1990) Die Zähmung der Monster. Auer, Heidelberg

\section{Intelligenz: Eine Frage der Vernetzung}

Intelligenz ist eng an Arbeitsstrukturen im Gehirn geknüpft: Je besser diese ausgebildet sind, desto leichter kann sich das Gehirn auf verschiedene Anforderungen einstellen. Das zeigt eine neue Studie der Universität Würzburg.

„Die Netzwerkarchitektur im Gehirn von Personen mit höheren Intelligenzwerten ähnelt bereits im Grundzustand der Architektur, die für verschiedene kognitive Anforderungen erforderlich ist", sagt Dr. Kirsten Hilger. Sie ist die Akademische Rätin und Leiterin der Forschergruppe "Networks of Behaviorand Cognition" am Lehrstuhl für Psychologie I der Julius-Maximilians-Universität Würzburg (JMU). Die neuronalen Grundlagen der menschlichen Intelligenz bilden einen Schwerpunkt ihrer Forschung. Gemeinsam mit ihrem Doktoranden Jonas A. Thiele und einem Team der Indiana University, Bloomington (USA) hat sie das von der Deutschen Forschungsgemeinschaft (DFG) geförderte Projekt durchgeführt.

Um den Unterschied zwischen funktionellen und strukturellen Gehirnnetzwerken zu erklären, greift die Neurowissenschaftlerin auf ein einfaches Bild zurück: Setzt man den Austausch von Informationen zwischen verschiedenen Hirnarealen mit dem Transport einer Ware von München nach Hamburg gleich, entspricht die Autobahn den strukturellen Gehirnverbindungen - je besser die Autobahn ausgebaut ist, desto schneller fließt der Verkehr. Der Verkehr seinerseits entspricht der funktionellen Verbindung des Gehirns. Diese funktionellen Verbindungen standen im Zentrum der aktuellen Studie.

Hierfür hat das Team auf die Daten von Untersuchungen an mehr als 800 Erwachsenen zugegriffen, die den Forschenden im Rahmen von internationalen "Data-Sharing" Initiativen zur Verfügunggestellt wurden.Die Teilnehmer*innen befanden sich entweder im Ruhestand oder mussten verschiedene Aufgaben bearbeiten. Währenddessen wurde mithilfe der funktionellen Magnetresonanztomografie (fMRT) die Aktivität ihrer Gehirnareale erfasst. „Mit den Bildern aus dem Magnetresonanztomografen konnten wir unsere Hypothese testen, dass ein höheres $\mathrm{Maß}$ an allgemeiner Intelligenz mit einer geringeren Rekonfiguration der Gehirnnetzwerke einhergeht", erklärt Hilger.

„Im Ruhezustand messen wir bei den Proban- den sozusagen den Grundverkehr, der immer fließt", erklärt Hilger. Eine externe kognitive Anforderung ist dann vergleichbar mit der Rushhour, einem Ferienwochenende oder einem Autocorso nach dem Gewinn der Weltmeisterschaft.

Hilger und ihr Team haben die Vorgänge sowohl im gesamten Gehirn analysiert als auch begrenzt auf verschiedene funktionelle Gehirnnetzwerke, von denen man weiß, dass sie mit bestimmten Gehirnfunktionen verbunden sind. Die Ergebnisse deuten darauf hin, dass die funktionellen Netzwerke von Menschen mit einem höheren Intelligenzwert beim Wechsel zwischen verschiedenen kognitiven Zuständen eine geringere Anpassung erfordern. Ihre Netzwerk-Architektur ist so gestaltet, dass der Wechsel nur geringe Umstellungenerfordert. Dieser Effekt trat unabhängig von der Art der zu bearbeitenden Aufgaben auf.

Dieses Ergebnis lässt den Schluss zu, dass Intelligenz eine Eigenschaft eines weit verteilten »Multitask-Gehirnnetzwerks« ist. Je intelligenter ein Mensch ist, desto besser ist die Netzwerkarchitektur seines Gehirns dafür geeignet, verschiedene kognitive Anforderungen zu erfüllen.

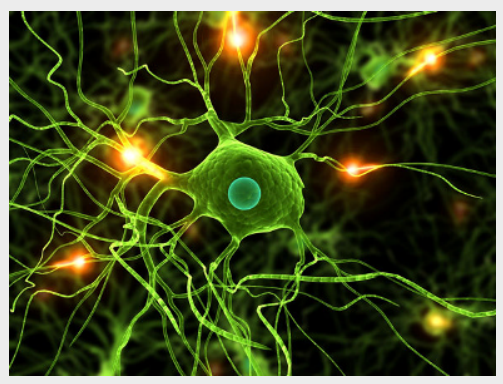

(c) Sebastian Kaulitzki / Fotolia

Originalpublikation: Multitask Brain Network Reconfiguration Is Inversely Associated with Human Intelligence. Jonas A Thiele, Joshua Faskowitz, Olaf Sporns, Kirsten

Hilger. Cerebral Cortex, https://doi.org/10.1093/cercor/bhab473

Quelle: Universität Würzburg (www.uniwuerzburg.de/aktuelles/[10.02.2022]) 